\title{
Magnetic resonance severity index assessed by T1-weighted imaging for acute pancreatitis: correlation with clinical outcomes and grading of the revised Atlanta classification - a narrative review
}

\author{
Giovanna Vacca ${ }^{1}$, Alfonso Reginelli ${ }^{1}$, Fabrizio Urraro ${ }^{1}$, Angelo Sangiovanni ${ }^{1}$, Federico Bruno ${ }^{2}$, \\ Ernesto Di Cesare ${ }^{3}$, Salvatore Cappabianca ${ }^{1}$, Angelo Vanzulli ${ }^{4}$
}

${ }^{1}$ Department of Precision Medicine, University of Campania “L. Vanvitelli”, Naples, Italy; ${ }^{2}$ Department of Biotechnology and Applied Clinical Sciences, University of L'Aquila, L'Aquila, Italy; ${ }^{3}$ Department of Life, Health and Environmental Sciences, University of L'Aquila, L'Aquila, Italy; ${ }^{4}$ Department of Radiology, University "La Statale” of Milan, Milan, Italy

Contributions: (I) Conception and design: A Vanzulli; (II) Administrative support: None; (III) Provision of study materials or patients: A Vanzulli, G Vacca; (IV) Collection and assembly of data: G Vacca; (V) Data analysis and interpretation: G Vacca; (VI) Manuscript writing: All authors; (VII) Final approval of manuscript: All authors.

Correspondence to: Alfonso Reginelli. Department of Precision Medicine, University of Campania “L. Vanvitelli”, Piazza Miraglia, 280138 Napoli (NA), Italy. Email: alfonsoreginelli@hotmail.com.

\begin{abstract}
Acute pancreatitis (AP) is a common disease that may involve pancreas and peripancreatic tissues with a prevalence of up to 50 per 100,000 individuals for year. The Atlanta classification was assessed for the first time in 1992 and modified in 2012 in order to describe morphological features of AP and its complications. AP can be morphologically distinguished in two main types: interstitial edematous pancreatitis (IEP) and necrotizing pancreatitis (NEP). This classification is very important because the presence of necrosis is directly linked to local or systemic complications, hospital stays and death. Magnetic resonance (MR) is very useful to characterize morphological features in AP and its abdominal complications. Particularly we would like to underline the diagnostic, staging and prognostic role of T1-weighted images with fat suppression that could be significant to assess many features of the AP inflammatory process and its complications (detection of the pancreatic contour, pancreatic necrosis, presence of haemorrhage). Signs of inflammatory and edema are instead observed by T1-weighted images. MR cholangiopancreatography (MRCP) is necessary to study the main pancreatic duct and the extrahepatic biliary tract and contrastenhancement magnetic resonance imaging (MRI) allows to assess the extent of necrosis and vascular injuries.
\end{abstract}

Keywords: Pancreatitis; imaging; Atlanta classification; magnetic resonance (MR); prognostic factors

Submitted Jun 14, 2020. Accepted for publication Nov 03, 2020.

doi: $10.21037 /$ gs-20-554

View this article at: http://dx.doi.org/10.21037/gs-20-554

\section{Introduction}

Acute pancreatitis (AP) is a common disease that may involve pancreas and peripancreatic tissues with a prevalence of up to 50 per 100,000 individuals for year $(1,2)$. The main causes of this inflammatory process are represented by biliary lithiasis and alcoholism (3). Diagnosis of AP is clinically suspected on the elevated serum amylase or lipase level associated especially with epigastric pain $(1,4,5)$.

^ ORCID: 0000-0003-4809-6235. 
Similarly to other inflammatory conditions, imaging plays an important role to confirm diagnosis and to detect complications $(1,5)$.

\section{The revised Atlanta classification}

The Atlanta classification was assessed for the first time in 1992 and modified in 2012 in order to describe morphological features of AP and its complications $(6,7)$.

AP can be morphologically distinguished in two main types: interstitial edematous pancreatitis (IEP) and necrotizing pancreatitis (NEP). This classification is very important because the presence of necrosis is directly related to local or systemic complications, hospital stays and death (7).

Acute NEP can be further stratified into three subcategories: combined pancreatic and peripancreatic necrosis (nearly $75 \%$ ), peripancreatic necrosis only (nearly $20 \%$ ), and pancreatic necrosis only (less than 5\%) (1,8-10).

IEP represents the most common clinical presentation and usually resolves in a few days. NEP is seen in about $5-10 \%$ of patients and its clinical management and therapy is more complex (7).

Both IEP and NEP might have local complications that we can consider in an early stage of disease if they happen within the first 4 weeks after the onset or later so after the first 4 weeks (9).

For IEP the acute complications are represented by acute pancreatic fluid collections (APFC) that can be single or multiple with homogeneous sterile content. If they do not resolve in a few weeks, they become pancreatic pseudocysts that are similar to APFC, but they show a wall of fibrous tissue (7).

For NEP within first 4 weeks we may discover acute necrotic collections (ANCs) that present irregular content of necrotic fatty tissue with pancreatic or extra-pancreatic debris (7).

After 4 weeks, ANCs could evolve into walled-off necrosis (WON) with enhancing inflammatory wall and can be sterile or infected (7). Respect to 1992, the revised Atlanta classification of 2012 divides patients into 3 categories based on severity: mild (no organ failure, no local or systemic complications), moderately severe (organ failure that resolves within $48 \mathrm{~h}$ and/or local or systemic complications without persistent organ failure), severe with persistent organ failure ( $>48 \mathrm{~h}$ ) (9). The new category assessed in 2012 is therefore moderately severe $\operatorname{AP}(9,11)$.

\section{Ultrasonography (US) imaging}

US represents mainly the first exam in the emergency setting that is executed after the clinical suspect diagnosis of AP. US shows often a regular pancreatic structure so it seems to be useful especially for the evaluation of the biliary tract $(8,12,13)$; unfortunately, in many cases it is not possible to visualize the distal bile duct because of the presence of the intestinal gas. US can also demonstrate the presence of biliary lithiasis (8).

According to us, US is more useful in monitoring the evolution of fluid collections and in guiding diagnostic and therapeutic interventions $(14,15)$.

\section{Computed tomography (CT) imaging}

CT acquisition protocol for the prompt diagnosis of AP consists at first of unenhanced acquisition to identify eventually also the most common cause of AP (biliary lithiasis) that are hyper-attenuating to bile and recognized on basal CT images only if calcified (10,16-18). The parenchymal phase (40 seconds after contrast administration) is useful to detect pancreatic necrosis areas because in this phase the healthy pancreatic tissue has the maximum enhancement $(16,18,19)$. The portal phase (70-80 seconds) extended to the whole abdomen offers the possibility to notice some local complications (venous thrombosis) (20). Three to five minutes later is possible to detect with confidence also if serious vascular complications caused by the regional inflammatory process such as pseudoaneurysms are ruptured or not: these walled sacs adjacent to the artery may bleed indeed into peritoneal cavity, retroperitoneal space and bowel (2,16,21-24).

The revised Atlanta classification is based on the use of contrast enhancement computed tomography (CECT) for the diagnosis, severity assessment and complications of AP (9). According to this classification, CECT should be performed at 5 to 7 days after the beginning of AP $(9,25)$. In IEP, pancreas may show enlargement due to inflammatory and edema but after contrast medium administration parenchyma is homogeneous and associated with mild peripancreatic fat stranding (7). In NEP lack of parenchymal enhancement indicates pancreatic necrosis. CECT may detect AFPCs that show a homogeneous and hypodense content with low attenuation values and without defined margins. Instead, ANCs are inhomogeneous with higher density and a well-defined wall $(7,26)$. WONs show solid and liquid density; the study of WON content 

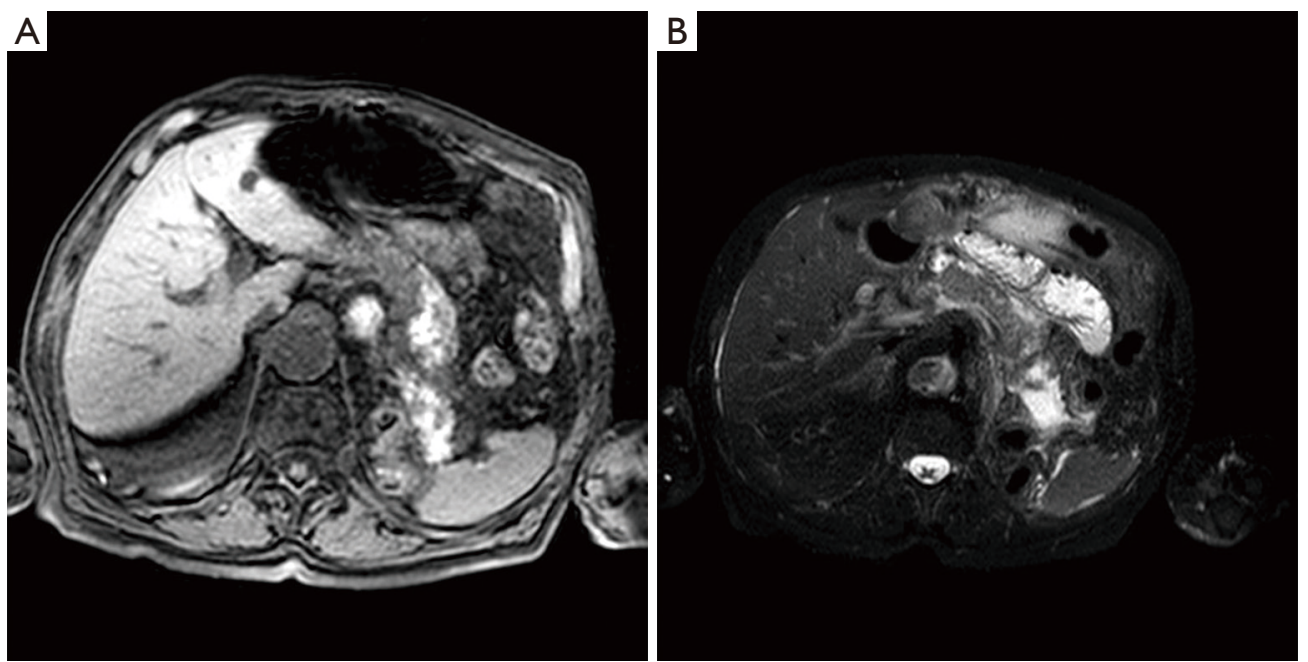

Figure 1 Acute edematous pancreatitis in a 67-year-old man. Axial non-enhanced magnetic resonance T1-weighted with fat-suppression image (A) and axial T2-weighted with fat-suppression image (B) show that the parenchyma of the pancreatic body and part of the tail is hypointense (A) and hyperintense (B) relative to the liver.

with CECT is useful to program further treatment as endoscopic or surgical necrosectomy (16). AP severity is principally related to presence of solid debris and fat necrosis and to the development of other local complications such as vascular injuries (splenic or portal vein thrombosis and pseudoaneurysm). The main inconvenient of CECT is the use of ionizing radiation; the iodinated contrast medium moreover is not considerable in patient with renal failure and previous severe reaction to iodinated contrast media (27-29).

\section{Magnetic resonance (MR) imaging}

MR is a multiparametric technique that is very useful to characterize and differentiate morphological features in $\mathrm{AP}$ and its local complications. Generally, MR protocol consists in T1-weighted fat-suppressed imaging singlebreath-hold gradient echo (GRE) sequence, T2-weighted fat-suppressed imaging with turbo spin-echo (TSE) or halfFourier acquisition single-shot turbo spin-echo (HASTE) sequence, diffusion-weighted imaging (DWI) and apparent diffusion coefficient (ADC) map, two- or three-dimensional MR cholangiopancreatography (MRCP) (HASTE heavily $\mathrm{T} 2$-weighted sequence) and dynamic contrast-enhanced (DCE) using T1-weighted acquisition $(3,30,31)$. All of these images are useful to assess $\mathrm{AP}$ severity, staging and complication $(32,33)$.

GRE T1-weighted images with fat suppression are fundamental first for the detection of the pancreatic contour in order to determine if there is a pancreatic enlargement or not (3). Moreover, these images allow to study the retroperitoneal fat and pancreatic necrosis but the most important feature that we can demonstrate is the presence of haemorrhage that is represented by hyperintensity in the pancreatic and peri-pancreatic areas (Figure 1).

Signs of inflammatory and edema are observed principally by T2-weighted images (Figure 1) (3).

MRCP is necessary to study the main pancreatic duct and the extrahepatic biliary tract especially to exclude duct leakage or disruption that is possible to notice in central gland necrosis (3).

DWI/ADC could reveal the presence of haemorrhage and necrosis. Some studies reported that DWI/ADC could be used also to detect infected collections with a greater diffusion restriction and lower ADC values (34).

Magnetic resonance imaging (MRI)-DCE allows to assess the extent of necrosis in the early phase of NEP because it has been demonstrated that MR severity index (MRSI) significantly correlates with Ranson score, CT severity index (CTSI), duration of hospitalization and clinical outcome $(35,36)$. Vascular injuries like pseudoaneurysm and phlebothrombosis could be also detected in this phase (1).

According to the revised Atlanta classification, acute fluid collections are hypointense on T1-weighted images and hyperintense in T2-weighted images if the content is serous (AFPCs) (3); on contrary if there is haemorrhage in T1-weighted, especially with fat suppression, is possible to 
observe hyperintensity (ANCs) (3). Necrotic tissues can be detected when typically spotted or with large hypointense areas on fat-suppressed T1-weighted images/T2-weighted images (although they may be hyperintense if liquefied) without any enhancement on DCE-MRI instead of the normal enhanced glandular tissue (3). After 4 weeks, WON would be distinguished from pancreatic pseudocysts by the presence in $\mathrm{T} 2$-weighted images of solid component and debris as hypointense material with variable shape or size. On contrary, a typical pancreatic pseudocyst would appear as a well-defined homogeneous fluid collection with smooth and symmetric walls that might enhance on DCE-MRI (37-40). We present the following article in accordance with the Narrative Review reporting checklist (available at http:// dx.doi.org/10.21037/gs-20-554).

\section{Methods}

We carefully searched in databases: PubMed/Medline, Web of Science, Google Scholar and Embase, from 2010 to 2020 to identify relevant studies, keywords including": Pancreatitis", "Imaging", "Atlanta Classification", "Magnetic Resonance", "Prognostic factors".

Eligible studies had to satisfy the following inclusion criteria: (I) recent studies (no longer than 10 years ago); (II) English articles; (III) studies focused on clinical experience of using MRI in the diagnosis and management of AP.

\section{Discussion}

First of all, in our clinical practice we have to consider that, in the emergency setting, US is very often requested for a preliminary study of the upper abdomen even if is often inconclusive: when pancreas can be visualized is regular in most of cases. According to surgeons' statement, ultrasound should be performed to determine the etiology of AP (biliary) in order to perform cholecystectomy for biliary pancreatitis (41). Surgery in the emergency setting is however uncommon: if the timing of laparoscopic cholecystectomy after an episode of mild acute biliary pancreatitis is controversial, it is well established that in severe pancreatitis delaying cholecystectomy after the resolution of inflammatory process should be preferred (42).

US is almost always followed by other diagnostic imaging, in most of cases by CECT.

Even if according to the revised Atlanta classification CECT should be performed at 5 to 7 days after the beginning of AP, it is often executed $72-96 \mathrm{~h}$ after the onset of symptoms especially to detect the extension of the peri-pancreatic necrosis (41). According to surgeons, the principal justification to perform MRCP should be to detect occult common bile duct stones in patients with unknown etiology (41).

In our clinical experience, even if CECT is mainly used for its accessibility and rapidity of execution, MR should be preferred, when possible, for many reasons.

If $\mathrm{MR}$ is available indeed, also in older patients or patients apparently unfit for being examined with $M R$, it can be however performed in most of cases because of its better capacity to assess AP findings severity of pancreatic and peripancreatic findings and complications.

On MRI T1-weighted and T2-weighted fat-suppressed sequences, when extra-pancreatic involvement is larger than the occurrence of fat stranding, is possible to detect spotted or patchy hypo-intensity areas within surrounding collections, that indicate pancreatic or peripancreatic necrosis (Figure 2). If it is possible to perform DCE-MR, these areas correspond to a lack of non-enhanced pancreatic parenchyma (43). At DCE-MRI based on the amount of pancreatic necrosis, it can be classified as less than $30 \%$ (mild), 30-50\% (moderate), and more than 50\% (severe) of the pancreatic gland $(3,44)$.

Patients with pancreatic necrosis, particularly with extensive necrosis (>30\%), should be examined with MRI because there is a well-established increase of mortality correlated directly not only to the presence but especially to the extent of pancreatic necrosis (45) that is possible to define properly only with MRI.

In addition, the clinical impact of performing MRI during the early phase of AP is related to the Acute Physiology and Chronic Health Evaluation (APACHE) II score, the systemic complication occurrence rate, hospitalization stay, and clinical outcome (46-49).

Above all, we would like to underline the diagnostic, staging and prognostic value of the T1-weighted images with fat sat suppression.

T1-weighted images with fat suppression is useful first to delineate properly pancreas and pancreatic borders (34). The enlarged pancreas in AIP is defined as anteriorposterior diameter $\geq 3 \mathrm{~cm}$ on axial plane (50).

The normal parenchyma shows high signal intensity in these images compared to the liver; on contrary decreased signal intensity of pancreas involved in the inflammatory process on T1-weighted imaging was established as not higher than that of liver (34).

MRI is superior to CT in detecting hemorrhagic AP 

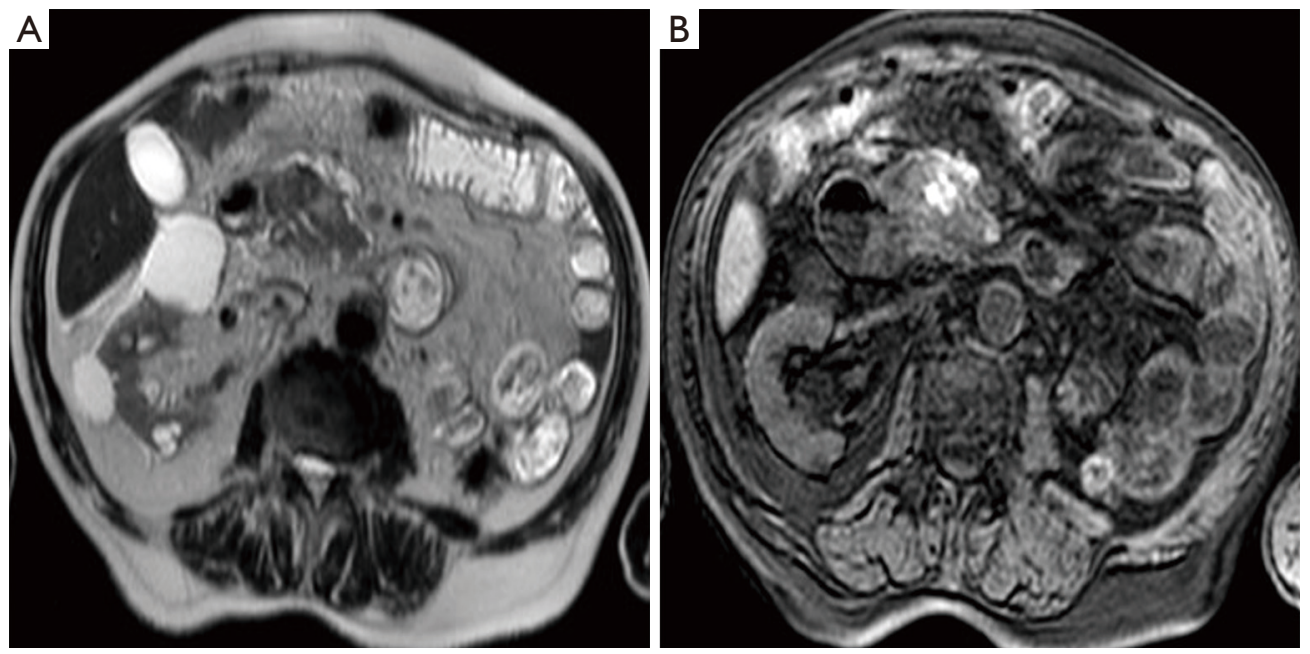

Figure 2 Acute edematous pancreatitis in a 53-year-old man. MR was obtained 4 days after the hospital admission. Axial magnetic resonance T2-weighted HASTE shows an area with intermediate signal in correspondence of the pancreatic head (A). T1-weighted with fatsuppression image demonstrates multiple patchy hemorrhagic foci (like "salt") (B).

because hemorrhage-related MRI signal changes over time (51-53). T1-weighted images with fat suppression shows the eventual presence of peri-pancreatic hemorrhage that might be secondary to the necrosis of peripancreatic tissue, which is represented by hyperintense patchy or extensive areas (43). The possibility to notice this feature is very remarkable because Martin and his group reported that elevated peripancreatic signal on fat suppressed T1-weighted images is linked to poor outcome in AP (54). Moreover, other local complications of AP such as pancreatic pseudocysts and peripancreatic vascular invasion, can also present hemorrhage (43).

In a recent study conducted by Xiao et al., it has been demonstrated that the presence of hemorrhage in AP (pancreatic hemorrhage and hemorrhagic fat necrosis) was common and that the hemorrhage severity index (HSI) was significantly associated with MRSI, higher organ dysfunction rate and longer hospitalization stay compared to those without hemorrhage $(3,55)$. This confirms the clinical importance to assess the presence of hemorrhage, almost exclusively with T1-weighted-fat suppressed images because it might correlate with a poorer prognosis of AP (3).

In a study conducted by Chi et al., MRI can detect also interfascial plane involvement in AP that was strongly correlated with the MRSI score because of its sensibility to assess fluid components (56).

MRI could be used to visualize the transverse mesocolon and it is important because some authors assessed that the mesocolon involvement on MRI was significantly correlated with the MRSI score and with the APACHE II score (57).

Therefore, as described also before, MR has the unique capability of allowing non-invasive evaluation of the pancreatic parenchyma, pancreatic ductal system, retroperitoneal soft tissue in the same exam (58). It has been reported that MRSI assessed by using 0.5 Tesla MR systems without contrast significantly correlated with CTSI, Ranson score, C-reactive protein levels, development of systemic complications, duration of hospitalization, and clinical outcome $(35,53,59)$.

The only complication that is really undetectable on unenhanced MRI is vascular involvement. Considering that is very uncommon to perform MR before CT in the clinical setting of AP, MR could be executed also without the injection of contrast medium because it however permits to demonstrate $\mathrm{AP}$ severity and staging.

Although CECT has been considered a traditional initial imaging modality for AP, it has a potential radiation risk when used in follow-up $(35,53)$. Compared with CECT, MRI is a multiparameter imaging that, thanks to its high contrast resolution, studies better soft tissue features without any risk of radiation that could be an advantage especially for AP patients that require multiple followup examinations. We have to consider patients that may develop recurrent AP indeed $(3,60)$.

Radiologist should choose MR also because imaging features of recurrent AP are less described in literature 
compared with chronic pancreatitis because this category is not included in the revised Atlanta classification (60).

It is already known that MR is limited by long times of acquisitions and expensiveness respect to US or CT. It requires patient cooperation and breath holding to avoid motion artefacts that could reduce the optimal visualization of the pancreas and its adjacent structures. But with the advance of new MRI techniques (phased-array coils, parallel imaging, triggering techniques) we could obtain encouraging results also in patients that are not able to cooperate with breath-hold instructions (34). Moreover, patients with AP are often young and require multiple follow-up examinations so preferring MR instead of CT in some patients would reduce their collective radiation dose significantly.

When possible and available, MR should be performed and preferred to CT in AP; the only aspect that in our opinion really could be considered as a potential weakness of MRI is its small field of view (3). Respect to CT, chest and pelvic evaluation cannot be obtained at one time but usually it is not necessary because it is demonstrated that AP staging strongly depends on the initial abdomen status (3).

\section{Conclusions}

Imaging plays a fundamental role in the initial identification of AP, allowing clinical evaluation and prognosis prediction during therapeutic management of patients $(16,61)$. According to us, MR is the most complete technique and should be preferred to CT during therapeutic management in the setting of AP for the detection and especially the staging of local complications. The correct differentiation between edematous and NEP (both in pancreatic or only peri-pancreatic presentations) and the appropriate characterization of complex fluid collections related to the pancreatic necrosis process are determining factors for a better management of the patients in order to assess prognosis stratification and definition of the best therapeutic strategy. During follow-up of AP (particularly for NEP) and for recurrent AP MR should be considered to avoid ionizing radiations.

\section{Acknowledgments}

Thanks to Valere Program by University of Campania "L. Vanvitelli".

Funding: None.

\section{Footnote}

Provenance and Peer Review: This article was commissioned by the Guest Editor (Antonio Barile) for the series "Multimodality Advanced Imaging and Intervention in Gland Diseases" published in Gland Surgery. The article has undergone external peer review.

Reporting Checklist: The authors have completed the Narrative Review reporting checklist. Available at http:// dx.doi.org/10.21037/gs-20-554

Conflicts of Interest: All authors have completed the ICMJE uniform disclosure form (available at http://dx.doi. org/10.21037/gs-20-554). The series "Multimodality Advanced Imaging and Intervention in Gland Diseases" was commissioned by the editorial office without any funding or sponsorship. The authors have no other conflicts of interest to declare.

Ethical Statement: The authors are accountable for all aspects of the work in ensuring that questions related to the accuracy or integrity of any part of the work are appropriately investigated and resolved.

Open Access Statement: This is an Open Access article distributed in accordance with the Creative Commons Attribution-NonCommercial-NoDerivs 4.0 International License (CC BY-NC-ND 4.0), which permits the noncommercial replication and distribution of the article with the strict proviso that no changes or edits are made and the original work is properly cited (including links to both the formal publication through the relevant DOI and the license). See: https://creativecommons.org/licenses/by-nc-nd/4.0/.

\section{References}

1. Xiao B, Zhang XM, Tang $W$, et al. Magnetic resonance imaging for local complications of acute pancreatitis: a pictorial review. World J Gastroenterol 2010;16:2735-42.

2. Reginelli A, Vacca G, Segreto T, et al. Can microvascular invasion in hepatocellular carcinoma be predicted by diagnostic imaging? A critical review. Future Oncol 2018;14:2985-94.

3. Xiao B, Xu HB, Jiang ZQ, et al. Current concepts for the diagnosis of acute pancreatitis by multiparametric magnetic resonance imaging. Quant Imaging Med Surg 
2019;9:1973-85.

4. Scialpi M, Reginelli A, D'Andrea A, et al. Pancreatic tumors imaging: An update. Int J Surg 2016;28 Suppl 1:S142-55.

5. Barile A, Brunese L, Giovagnoni A. Gland diseases: new perspectives in diagnostic radiology. Gland Surg 2019;8:S126-9.

6. Banks PA, Bollen TL, Dervenis C, et al. Classification of acute pancreatitis--2012: revision of the Atlanta classification and definitions by international consensus. Gut 2013;62:102-11.

7. Dhaka N, Samanta J, Kochhar S, et al. Pancreatic fluid collections: What is the ideal imaging technique? World J Gastroenterol 2015;21:13403-10.

8. Cunha EF, Rocha Mde S, Pereira FP, et al. Walled-off pancreatic necrosis and other current concepts in the radiological assessment of acute pancreatitis. Radiol Bras 2014;47:165-75.

9. Ignatavicius P, Gulla A, Cernauskis K, et al. How severe is moderately severe acute pancreatitis? Clinical validation of revised 2012 Atlanta Classification. World J Gastroenterol 2017;23:7785-90

10. Grassedonio E, Toia P, La Grutta L, et al. Role of computed tomography and magnetic resonance imaging in local complications of acute pancreatitis. Gland Surg 2019;8:123-32.

11. Addeo G, Beccani D, Cozzi D, et al. Groove pancreatitis: a challenging imaging diagnosis. Gland Surg 2019;8:S178-87.

12. Reginelli A, Urraro F, di Grezia G, et al. Conventional ultrasound integrated with elastosonography and B-flow imaging in the diagnosis of thyroid nodular lesions. Int J Surg 2014;12 Suppl 1:S117-22.

13. Carrafiello G, Fontana F, Cotta E, et al. Ultrasoundguided thermal radiofrequency ablation (RFA) as an adjunct to systemic chemotherapy for breast cancer liver metastases. Radiol Med 2011;116:1059-66.

14. Scotto di Santolo M, Massimo C, Tortora G, et al. Clinical value of high-resolution (5-17 MHz) echo-color Doppler (ECD) for identifying filling materials and assessment of damage or complications in aesthetic medicine/surgery. Radiol Med 2019;124:568-74.

15. Belfiore G, Belfiore MP, Reginelli A, et al. Concurrent chemotherapy alone versus irreversible electroporation followed by chemotherapy on survival in patients with locally advanced pancreatic cancer. Med Oncol 2017;34:38.

16. Takahashi N, Papachristou GI, Schmit GD, et al. CT findings of walled-off pancreatic necrosis (WOPN): differentiation from pseudocyst and prediction of outcome after endoscopic therapy. Eur Radiol 2008;18:2522-9.

17. Belfiore MP, Reginelli A, Maggialetti N, et al. Preliminary results in unresectable cholangiocarcinoma treated by CT percutaneous irreversible electroporation: feasibility, safety and efficacy. Med Oncol 2020;37:45.

18. Reginelli A, Vanzulli A, Sgrazzutti C, et al. Vascular microinvasion from hepatocellular carcinoma: CT findings and pathologic correlation for the best therapeutic strategies. Med Oncol 2017;34:93.

19. Arrigoni F, Barile A, Zugaro L, et al. CT-guided radiofrequency ablation of spinal osteoblastoma: treatment and long-term follow-up. Int J Hyperthermia 2018;34:321-7.

20. Reginelli A, Capasso R, Ciccone V, et al. Usefulness of triphasic CT aortic angiography in acute and surveillance: Our experience in the assessment of acute aortic dissection and endoleak. Int J Surg 2016;33:S76-84.

21. Mazzei MA, Nardone V, Di Giacomo L, et al. The role of delta radiomics in gastric cancer. Quant Imaging Med Surg 2018;8:719-21.

22. Reginelli A, Vacca G, Zanaletti N, et al. Diagnostic value/performance of radiological liver imaging during chemoterapy for gastrointestinal malignancy: A critical review. Acta Biomedica 2019;90:51-61.

23. Iacobellis F, Laccetti E, Tamburrini S, et al. Role of multidetector computed tomography in the assessment of pancreatic injuries after blunt trauma: a multicenter experience. Gland Surg 2019;8:184-96.

24. Angileri SA, Gorga G, Tortora S, et al. Biliary injuries after pancreatic surgery: interventional radiology management. Gland Surg 2019;8:141-9.

25. Nardone V, Tini $\mathrm{P}$, Nioche $\mathrm{C}$, et al. Texture analysis as a predictor of radiation-induced xerostomia in head and neck patients undergoing IMRT. Radiol Med 2018;123:415-23.

26. Sheafor DH, Kovacs MD, Burchett $\mathrm{P}$, et al. Impact of low-kVp scan technique on oral contrast density at abdominopelvic CT. Radiol Med 2018;123:918-25.

27. Anderson SW, Lucey BC, Varghese JC, et al. Accuracy of MDCT in the diagnosis of choledocholithiasis. AJR Am J Roentgenol 2006;187:174-80.

28. Molinelli V, Iosca S, Duka E, et al. Ability of specific and nonspecific signs of multidetector computed tomography (MDCT) in the diagnosis of blunt surgically important bowel and mesenteric injuries. Radiol Med 2018;123:891-903.

29. Giurazza F, Corvino F, Cavaglia E, et al. Arterial embolizations with microvascular plug in extracranial and intracranial districts: technical results. Radiol Med 
2018;123:236-43.

30. Petralia G, Padhani AR, Pricolo P, et al. Wholebody magnetic resonance imaging (WB-MRI) in oncology: recommendations and key uses. Radiol Med 2019;124:218-33.

31. Nardone V, Reginelli A, Scala F, et al. MagneticResonance-Imaging Texture Analysis Predicts Early Progression in Rectal Cancer Patients Undergoing Neoadjuvant Chemoradiation. Gastroenterol Res Pract 2019;2019:8505798.

32. Reginelli A, Di Grezia G, Izzo A, et al. Imaging of adrenal incidentaloma: our experience. Int J Surg 2014;12 Suppl 1:S126-31.

33. La Marra A, Quarchioni S, Ferrari F, et al. 640-Slice CT Measurement of Superior Orbital Fissure as Gateway for Light into the Brain: Statistical Evaluation of Area and Distance. PLoS One 2016;11:e0162940.

34. Busireddy KK, AlObaidy M, Ramalho M, et al. Pancreatitis-imaging approach. World J Gastrointest Pathophysiol 2014;5:252-70.

35. Arvanitakis M, Koustiani G, Gantzarou A, et al. Staging of severity and prognosis of acute pancreatitis by computed tomography and magnetic resonance imaging-a comparative study. Dig Liver Dis 2007;39:473-82.

36. Islim F, Salik AE, Bayramoglu S, et al. Non-invasive detection of infection in acute pancreatic and acute necrotic collections with diffusion-weighted magnetic resonance imaging: preliminary findings. Abdom Imaging 2014;39:472-81

37. Macari M, Finn ME, Bennett GL, et al. Differentiating pancreatic cystic neoplasms from pancreatic pseudocysts at MR imaging: value of perceived internal debris. Radiology 2009;251:77-84.

38. Miller FH, Keppke AL, Dalal K, et al. MRI of pancreatitis and its complications: part 1, acute pancreatitis. AJR Am J Roentgenol 2004;183:1637-44.

39. Liu $Y$, Wang $Y$, Tang $W$, et al. Multiparametric MR imaging detects therapy efficacy of radioactive seeds brachytherapy in pancreatic ductal adenocarcinoma xenografts. Radiol Med 2018;123:481-8.

40. Galia M, Agnello F, Sparacia G, et al. Evolution of indeterminate hepatocellular nodules at Gd-EOBDPTA-enhanced MRI in cirrhotic patients. Radiol Med 2018;123:489-97.

41. Leppäniemi A, Tolonen M, Tarasconi A, et al. 2019 WSES guidelines for the management of severe acute pancreatitis. World J Emerg Surg 2019;14:27.

42. Dedemadi G, Nikolopoulos M, Kalaitzopoulos I, et al.
Management of patients after recovering from acute severe biliary pancreatitis. World J Gastroenterol 2016;22:7708-17.

43. Xiao B, Zhang XM. Magnetic resonance imaging for acute pancreatitis. World J Radiol 2010;2:298-308.

44. Balthazar EJ. Acute pancreatitis: assessment of severity with clinical and CT evaluation. Radiology 2002;223:603-13.

45. Mortele KJ, Wiesner W, Intriere L, et al. A modified CT severity index for evaluating acute pancreatitis: improved correlation with patient outcome. AJR Am J Roentgenol 2004;183:1261-5.

46. Tang G, Shak S, Paik S, et al. Comparison of the prognostic and predictive utilities of the 21-gene Recurrence Score assay and Adjuvant! for women with node-negative, ER-positive breast cancer: results from NSABP B-14 and NSABP B-20. Breast Cancer Res Treat 2011;127:133-42.

47. Xie CL, Zhang M, Chen Y, et al. Spleen and splenic vascular involvement in acute pancreatitis: an MRI study. Quant Imaging Med Surg 2018;8:291-300.

48. Sun H, Zuo HD, Lin Q, et al. MR imaging for acute pancreatitis: the current status of clinical applications. Ann Transl Med 2019;7:269.

49. Tonolini M, Di Pietro S. Diffusion-weighted MRI: new paradigm for the diagnosis of interstitial oedematous pancreatitis. Gland Surg 2019;8:197-206.

50. Zhang XM, Feng ZS, Zhao QH, et al. Acute interstitial edematous pancreatitis: Findings on non-enhanced MR imaging. World J Gastroenterol 2006;12:5859-65.

51. Balci NC, Bieneman BK, Bilgin M, et al. Magnetic resonance imaging in pancreatitis. Top Magn Reson Imaging 2009;20:25-30.

52. Robinson PJA, Sheridan MB. Pancreatitis: computed tomography and magnetic resonance imaging. Eur Radiol 2000;10:401-8.

53. Arvanitakis M, Delhaye M, De Maertelaere V, et al. Computed tomography and magnetic resonance imaging in the assessment of acute pancreatitis. Gastroenterology 2004;126:715-23.

54. Martin DR, Karabulut N, Yang M, et al. High signal peripancreatic fat on fat-suppressed spoiled gradient echo imaging in acute pancreatitis: preliminary evaluation of the prognostic significance. J Magn Reson Imaging 2003;18:49-58.

55. Tang MY, Chen TW, Bollen TL, et al. MR imaging of hemorrhage associated with acute pancreatitis. Pancreatology 2018;18:363-9.

56. Chi XX, Chen TW, Huang XH, et al. Magnetic resonance 
imaging of retroperitoneal interfascial plane involvement in acute pancreatitis. Quant Imaging Med Surg 2016;6:250-8.

57. Chi XX, Zhang XM, Chen TW, et al. The normal transverse mesocolon and involvement of the mesocolon in acute pancreatitis: an MRI study. PloS One 2014;9:e93687.

58. Manikkavasakar S, AlObaidy M, Busireddy KK, et al. Magnetic resonance imaging of pancreatitis: an update. World J Gastroenterol 2014;20:14760-77.

59. Stimac D, Miletić D, Radić M, et al. The role of nonenhanced magnetic resonance imaging in the early

Cite this article as: Vacca G, Reginelli A, Urraro F, Sangiovanni A, Bruno F, Di Cesare E, Cappabianca S, Vanzulli A. Magnetic resonance severity index assessed by T1-weighted imaging for acute pancreatitis: correlation with clinical outcomes and grading of the revised Atlanta classification-a narrative review. Gland Surg 2020;9(6):2312-2320. doi: 10.21037/ gs-20-554 assessment of acute pancreatitis. Am J Gastroenterol 2007;102:997-1004.

60. Avanesov M, Löser A, Smagarynska A, et al. Clinicoradiological comparison and short-term prognosis of single acute pancreatitis and recurrent acute pancreatitis including pancreatic volumetry. PloS One 2018;13:e0206062.

61. Wan J, Fang J, Li G, et al. Pancreas allograft biopsies procedure in the management of pancreas transplant recipients. Gland Surg 2019;8:794-8. 